\section{A reduction in ECS-produced amnesia through cooling*}

\author{
SARAH L. SMITH and JAMES R. MISANIN† \\ Susquehanna University, Selinsgrove, $\mathrm{Pa} .17870$
}

Ninety-six rats were trained on a passive avoidance task and then given one of six treatments to determine how ECS affects retention when followed by hypothermia. Although ECS alone produced some degree of retention impairment, when followed by hypothermia, it had no effect on retention. These results were interpreted in terms of hypothermia-induced attenuation of sensory input following ECS and lend support to a memory-retrieval-failure interpretation of ECS-produced amnesia.

Hypothermia induced in rodents immediately after training on a one-trial passive avoidance task has been shown to induce a retention deficit similar to that produced by other so-called amnesic agents (Beitel \& Porter, 1968; Riccio, Gaebelein, \& Cohen, 1968; Riccio, Hodges, \& Randall, 1968). A more recent finding, however, is that the hypothermia per se is not a sufficient condition for such deficits. Misanin \& Hoover (1971) found that a relatively rapid rate of recovery from hypothermia was a major determinant of hypothermia-induced amnesia. They suggested that the amnesic-like effect of hypothermia and possibly that of other so-called amnesic agents is due, not to disruption of memory consolidation, but, rather, to an inability to retrieve stored material as a consequence of the input following the amnestic treatment. It was assumed that the slow rate of recovery from hypothermia maintained a low sensory input and, hence, reduced the probability of retrieval failure. Thus, they hypothesized that the neuroelectrical activity specific to a learned event plays a catalytic role in memory storage and that disruption of this activity slows the storage process, thereby increasing the time over which additional inputs can scramble the memory code to produce retrieval difficulty.

The present study sought to test this hypothesis by inducing hypothermia in rats immediately after an electroconvulsive shock (ECS) given following passive avoidance training. It was reasoned that if ECS simply slows the memory storage process by disrupting the neuroelectrical activity specific to this training, then a state of reduced sensory input following ECS, brought about and maintained by a

*This research was supported by PHS Grant MH-17207 from the National Institute of Mental Health.

+ Requests for reprints should be sent to the second author. reduction in body temperature, should attenuate, if not eliminate, the amnesic-like effect of ECS.

\section{METHOD}

The data are from 48180 - to $290-\mathrm{g}$ male and 48 150- to $225 \mathrm{~g}$ female albino rats of the Wistar strain born and reared in the Susquehanna University animal colony. All animals were individually housed in a $24^{\circ}-26^{\circ} \mathrm{C}$ room and were given free access to water and $10 \mathrm{~g}$ of food daily.

The step-down apparatus used for passive avoidance training has been previously described (Misanin \& Hoover, 1971). It consisted of a $20 \times 20$ in. grid floor enclosed by 24-in.-high transparent acrylic walls and a $4 \times 5 \times 3$ in. platform located at its center. The floor of the apparatus was connected to a Harvard Instrument shock generator and scrambler (Model 3121 ) set at $1.6 \mathrm{~mA}$.

A Lafayette Instrument Co. shocker (Model A-615 B) set at $600 \mathrm{~V}$ and $10 \mathrm{~K} \mathrm{ohms}$ was used to deliver a $0.5 \mathrm{sec}$ ECS through modified alligator clips attached to S's ears.

A Forma Temp Jr. bath and circulator (Model 2095) set to maintain tap water at $0.5^{\circ}-3^{\circ} \mathrm{C}$ was used to induce hypothermia. Hypothermia was induced by restraining the animal in a 2 -in.-diam immersing it up to the ears in the water for $10 \mathrm{~min}$. A Yellow Springs Tele-Thermometer (Model 43 TD) and a modified No. 401 probe were used in recording colonic temperatures (cf. Misanin \& Hoover, 1971).

On the 2 days preceding training, each animal received three daily $3-\mathrm{min}$ handling treatments followed by $3 \mathrm{~min}$ of earclip adaption in its home cage; treatments were given at $30-\mathrm{min}$ intervals. On the day of training, $S$ was weighed and given three trials in the step-down apparatus with earclips on the central platform and allowing it to step down to the grid floor where it remained for $5 \mathrm{sec}$. The criterion for a step-down response was having all four perforated acrylic cylinder and attached. A trial consisted of placing S feet on the grid floor. After each of the first two trials, $S$ was removed from the apparatus to its home cage for approximately $30 \mathrm{~min}$. The third, or training, trial differed from the others in poststep-down treatment of $\mathrm{S}$; groups were also differentiated on this basis.

Four groups of eight males and eight females each received a 5 -sec footshock (FS) upon stepping from the platform on the training trial. One of these (FS-ECS-C) was given ECS simultaneously with FS offset and then immediately given the hypothermal treatment. After removal from the water, colonic temperature was recorded and $S$ was placed in a wire mesh cage in a $18^{\circ}-20^{\circ} \mathrm{C}$ room for $50 \mathrm{~min}$. After this time, S's temperature was again recorded and $S$ returned to its home cage. A second group (FS-ECS-NC) received treatment identical to the FS-ECS-C group, except that animals of this group were suspended in an empty tank instead of immersed in cold water. The two remaining $\mathrm{FS}$ groups did not receive ECS. One of these (FS-NECS-C), however, was otherwise treated the same as the FS-ECS-C group, and the other (FS-NECS-NC), the same as the FS-ECS-NC group. The remaining 32 Ss received no FS upon their descent from the platform. One group (NFS-ECS-C) of 8 males and 8 females received ECS $5 \mathrm{sec}$ after its descent, followed immediately by the hypothermal treatment; the remaining group (NFS-NECS-NC) was simply suspended in an empty tank and received the posttraining temperature recording treatments.

Eight females and three males died as a result of the hypothermal treatment and were replaced. Of these, nine Ss were from the FS-NECS-C group and one each was from the other hypothermal groups.

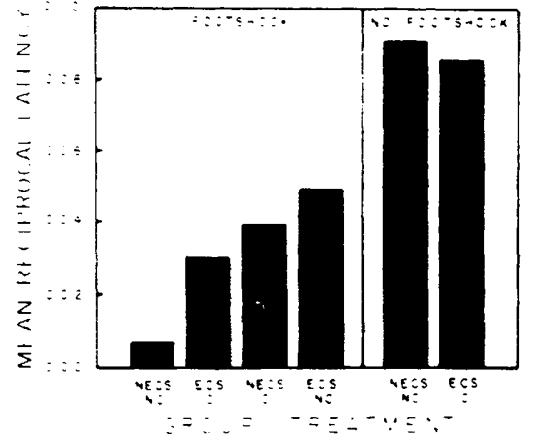

Fig. 1. Mean reciprocal step-down latencies of the footshock and no-footshock groups as a function of posttraining treatment. The ECS refers to electroconvulsive shock, the $\mathrm{C}$ to the hypothermal treatment, immersion in cold water, and $\mathrm{N}$ to the absence of the treatment. 
Table 1

Immediate (0-Min) and Delayed (50-Min) Postimmersion Colonic Temperature (Degrees Centigrade)

Group Treatment

\begin{tabular}{rccccccc} 
& \multicolumn{4}{c}{ Footshock } & & \multicolumn{2}{c}{ No Footshock } \\
\cline { 2 - 5 } & ECS-C & NECS-C & ECS-NC & NECS-NC & & ECS-C & ECS-NC \\
\hline O M in & 19.2 & 17.6 & 34.2 & 35.1 & & 18.9 & 34.0 \\
$50 \mathrm{Min}$ & 25.9 & 20.0 & 34.1 & 34.6 & & 25.7 & 34.0 \\
\hline
\end{tabular}

All animals were tested for retention of the passive avoidance task $24 \mathrm{~h}$ after training. A test trial was the same as a pretraining trial. During training and testing, time to step down from the platform (latency) was recorded to the nearest .1 sec. If, during testing, an animal failed to descend to the grid floor within $10 \mathrm{~min}$, the trial was terminated and $\mathrm{S}$ assigned a latency of $600.0 \mathrm{sec}$

RESULTS AND DISCUSSION

The latency scores of each animal for training and testing were converted to speed measures (reciprocal latency) and analyzed. A 2 by 6 factorial analysis of variance, with sex and group being the factors under consideration, showed no significant sex or group differences during training (Fs < 1.0). A preliminary analysis of the test data also failed to show a within-group sex effect. Thus, sex was ignored in the presentation of the results (Fig. 1) and in subsequent group comparisons.

The test data from the FS groups (left panel, Fig. 1) were analyzed as a 2 by 2 factorial design, with presence and absence being the variation in ECS and hypothermia. An overall group effect $(F=3.32, \mathrm{df}=3,60, \mathrm{p}<.05)$ and ECS by Hypothermia interaction $(\mathrm{F}=6.72, \mathrm{df}=1,60, \mathrm{p}<.025)$ were obtained. A one-way analysis of variance on the data from the NFS groups (right panel, Fig. 1), on the other hand, showed no significant difference between groups, suggesting that the combined ECS-hypothermia treatment had no proactive effect on step-down latency. For individual comparisons between FS and NFS groups, the mean squares within groups of these two analyses were pooled to derive the denominator of the $t$ ratio; the mean square from the 2 by 2 analysis only was used in this derivation for the comparisons between FS groups. These comparisons, made at the .05 level of significance, indicated that the FS-ECS-NC group showed the typical ECS-induced retention impairment, differing from the FS-NECS-NC group and failing to differ from both NFS groups. The FS-ECS-C group, on the other hand, failed to differ from the FS-NECS-NC group and did differ from the NFS groups, indicating that hypothermia eliminated the amnesic-like effect of ECS. The FS-NECS-C group, however, showed moderate retention impairment differing from only the NFS-NECS-NC group.

The present results tend to support the hypothesis that amnesic agents produce their effects by slowing rather than disrupting the memory storage process (Misanin \& Hoover, 1971). According to this notion, slowing of the memory process simply provides ample opportunity for additional inputs into the system to interfere with the encoding of memory, which, in turn, increases the probability of retrieval difficulty. In the present study, for example, an attenuation of input following ECS, brought about and maintained by a reduction in body temperature, could explain the lack of amnesic-like behavior in the FS-ECS-C Ss. An interpretation of ECS-induced amnesia in terms of disruption of the storage process cannot account for this result (e.g., McGaugh, 1966).

A puzzling finding, and one that appears incongruous with the suggested retrieval-failure explanation, is that the FS-NECS-C Ss, even though they recovered from hypothermia at a lower rate than the FS-ECS-C Ss (see Table 1), showed some degree of retention impairment. There are at least two explanations of this retention deficit that do not conflict with the retrieval-failure hypothesis.
One focuses on the fact that animals in the FS-ECS-C group were in an ECS-induced comatose state at the time they were immersed, whereas those in the FS-NECS-C group were not. Thus, sensory input associated with restraint and cold-water immersion prior to unconsciousness induced by a body temperature drop may have been sufficient to produce the slight retention deficit observed in the FS-NECS-C Ss. The second explanation concerns the mortality rate of animals subjected to the hypothermal treatment. Of the 11 animals that succumbed during treatment, 9 were in the FS-NECS-C group. Thus, the members of this group may be considered more homogeneous than those of the other experimental groups. This is, in fact, suggested in the within-group variability of scores. The truncated range (extremes excluded) for each group, as ordered from left to right in Fig. 1 , was .003-.019, .004-.072, $.012-.067, .004-.125, .027-.182$, and $.005-.333$, respectively. The 14 surviving animals of the FS-NECS-C group, then, may also have been less susceptible to stress than a completely random sample of Ss. It is possible, therefore, that the FS situation was also a less stressful experience for this somewhat select group than for the random sample of Ss in the FS-NECS-NC group, so that what appears to be amnesia for $F S$ in the FS-NECS-C Ss reflects, simply, less fear conditioned by FS.

\section{REFERENCE}

BEITEL, R. E., \& PORTER, P. B. Deficits in short- and long-term retention and impairments in learning induced by severe hypothermia in mice. Journal of Comparative \& Physiological Psychology, $1968,66,53-59$.

McGAUGH, J. L. Time dependent processes in memory storage. Science, 1966,153 , 1351-1358.

MISANIN, J. R., \& HOOVER, M. Recovery rate as a determinant of the amnesic-like effect of hypothermia Physiology \& Behavior, 1971, 6, 689-693.

RICCIO, D. C. GAEBELEIN, C.. \& COHEN, P. Some behavioral aspects of retrograde amnesia produced by hypothermia. Physiology \& Behavior, $1968,3,973-976$.

RICCIO, D. C., HODGES, L. A., \& RANDALL, P. K. Retrograde amnesia produced by hypothermia in rats. Journal of Comparative \& Physiological Psychology, 1968, 66, 618-622. 